\title{
An alternative path to reduce neuroinflammation
}

The discovery in the nervous system of an alternative route for arachidonic acid (AA) production - from which cyclooxygenases (COXs) synthesize neuroinflammatory prostaglandins - might offer a safer way of suppressing the inflammation that is characteristic of neurodegenerative disorders such as Parkinson's disease. Phospholipase A2 (PLA2) enzymes have been thought to be the main source of AA production, but Nomura et al. now report in Science that AA can be generated in the brain from the endocannabinoid 2-arachidonoylglycerol (2-AG) by

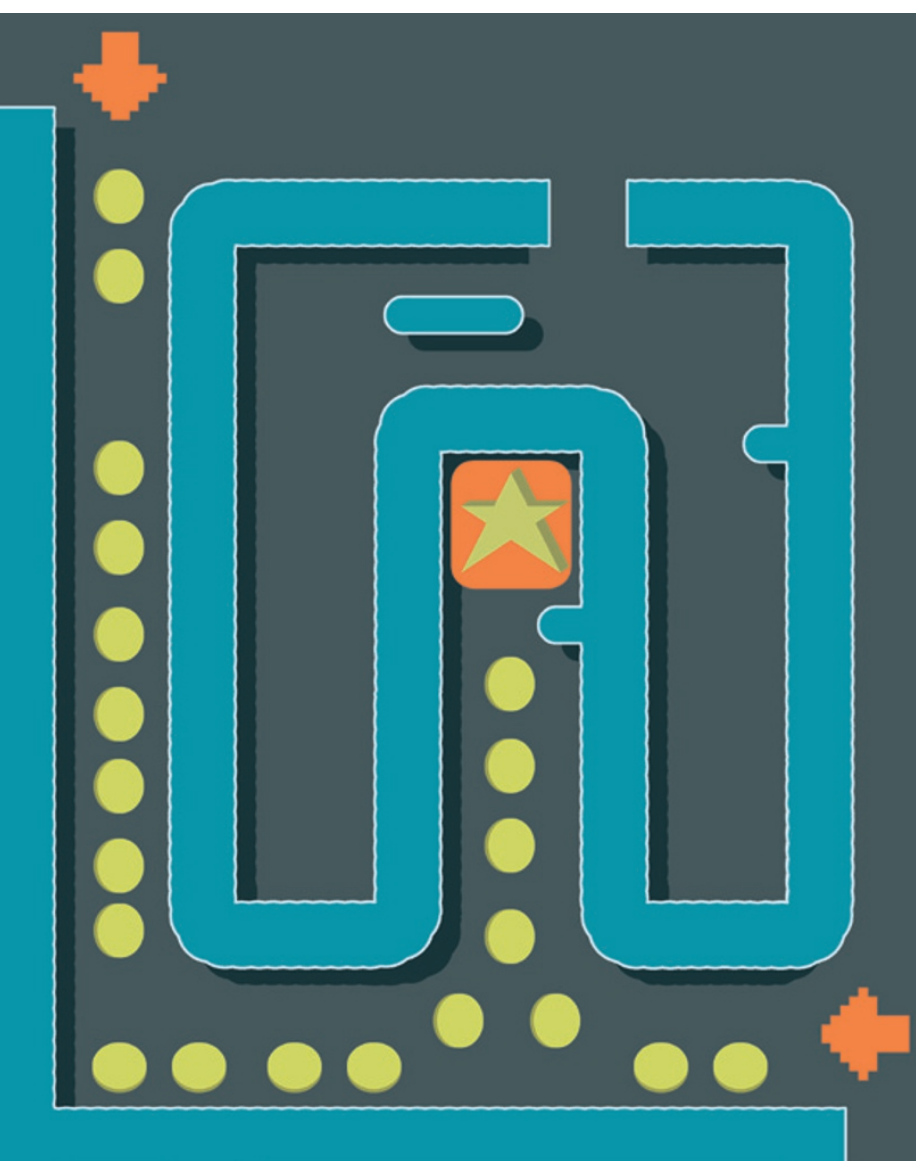

monoacylglycerol lipase (MAGL), suggesting that MAGL inhibition could be a novel anti-inflammatory strategy.

The existence of non-PLA2mediated mechanisms of AA production had been indicated by the previous finding that AA levels in the brain are unaltered in mice lacking cytosolic PLA2. In addition, brain levels of AA and several downstream AA-derived eicosanoids were decreased in mice lacking the gene that encodes MAGL $\left(\mathrm{Mgll}^{-/-}\right.$mice $)$and in mice treated with the MAGL-selective inhibitor JZL184, alongside increased levels of the 2-AG substrate.

To investigate the relevance of MAGL in neuroinflammation, the authors compared wild-type mice with $\mathrm{Mgll}^{-/-}$or JZL184-treated mice to study the effect of the proinflammatory agent lipopolysaccharide (LPS) on lipid levels in the brain. MAGL-deficient animals displayed impaired eicosanoid production as well as suppressed cytokine levels and a lack of microglial activation in response to LPS. Antagonizing cannabinoid receptor 1 or cannabinoid receptor 2 could not reverse these effects, indicating that decreased levels of AA, rather than increased endocannabinoid signalling, were responsible. Cytosolic PLA2 also contributed, albeit to a lesser extent than MAGL, to the AA pool available for the production of prostaglandins in the brain.

Given the effect of MAGL deficiency on LPS-induced eicosanoid production in the brain, the authors next investigated the potential consequences of its absence in a mouse model of Parkinson's disease induced by 1-methyl-4-phenyl-1,2,3,6-tetrahydropyridine (MPTP). $\mathrm{Mgll}^{-/-}$mice or JZL184-treated mice were protected from MPTP-induced loss of dopaminergic neurons in the substantia nigra and failed to show an MPTP-induced increase in levels of AA, prostaglandins and proinflammatory cytokines in the brain.

Identical results were seen following COX inhibition in MPTP-treated mice; indeed, COX inhibitors are known to confer neuroprotection in rodent models of inflammationmediated neurodegenerative disorders. Their pharmacological use in such disorders, however, is hampered by their gastrointestinal toxicity. Importantly, the authors found that although MAGL also contributed substantially to the production of prostaglandins from AA in the liver and lungs, cytoplasmic PLA2 regulated AA production in the gut and spleen. This suggests that the gastric haemorrhaging associated with the use of COX inhibitors might be avoided by instead targeting MAGL inhibition, which was supported by studies with JZL184.

So, this report has uncovered not only an alternative means of regulating prostaglandin production in the nervous system but also a potentially safer way to reduce inflammation in neurodegenerative diseases. However, as the authors caution, the possibility that MAGL inhibitors might be associated with mild physical dependence (owing to downregulation of cannabinoid receptors) will be important to consider in establishing the translational potential of these findings.

Katrin Legg

ORIGINAL RESEARCH PAPER Nomura, D. K. et al Endocannabinoid hydrolysis generates brain prostaglandins that promote neuroinflammation. Science 20 Oct 2011 (doi: 10.1126/science.1209200) 